\title{
Incidencia de lesiones en la práctica deportiva del motocross
}

\author{
Incidence of injuries in the sport practice of motocross
}

\author{
***Flavio Boechat de Oliveira, *Mariah Dias Morgado Pereira, *Angélica de Mendonça Nunes, \\ $* *$ Rafael Barreto, ***Frederico Pecorone Matos, ****Rodrigo Gomes de Souza Vale
}

Oliveira, F.B., Dias Morgado, M., de Mendonça, A., Barreto, R., Pecorone, F. \& Vale, R. (2017). Incidencia de lesiones en la práctica deportiva del motocross. Revista Ciencias de la Actividad Física UCM, No 18(2) julio-diciembre, 1-9.

\begin{abstract}
RESUMEN
Antecedentes: Estudiar la incidencia de lesiones que involucran a atletas profesionales de Motocross de diferentes categorías en niveles de carreras estatales, nacionales e internacionales y evaluar las lesiones más comunes dentro de los atletas de Motocross y las áreas más frecuentemente afectadas por la práctica del deporte. Métodos: El estudio incluyó un cuestionario respondido por 45 atletas entre 16 y 46 años de edad, hombres y mujeres, de diferentes nacionalidades y lugares de nacimiento, en carreras para el Campeonato de Motocross de Brasil. Resultados: Las fracturas son las lesiones más comunes entre los corredores de Motocross (17.55 \%), seguido de dislocaciones (13.05\%). Los hombros y las rodillas son los sitios anatómicos más afectados. Las pruebas de chi- cuadrado mostraron que el diagnóstico tiene una relevancia significativa sobre la influencia y la definición del tratamiento de las lesiones $(\mathrm{p}=0.001)$. El diagnóstico tuvo significación estadística en relación con el regreso de los corredores a los deportes con el mismo nivel físico anterior a la lesión $(\mathrm{p}=0.001)$. Conclusión: Los hombros fueron los más afectados por las lesiones y las fracturas la lesión más común en la práctica de Motocross y debido a su naturaleza de alto impacto, el Motocross tiene una alta tasa de lesiones.
\end{abstract}

\section{PALABRAS CLAVE}

Lesiones, lesiones deportivas, medicina deportiva, actividad física.

\begin{abstract}
Background: To study the incidence of injuries involving professional Motocross athletes from different categories in state, national and international racing levels and to evaluate the most common injuries within Motocross athletes and the most frequently affected areas by the sport's practice. Methods: The study included a questionnaire answered by 45 athletes aged between 16 and 46 years old, male and female, of different nationalities and places of birth, racing for the Brazilian Motocross Championship. Results: Fractures are the most common injuries among Motocross racers (17.55\%), followed by dislocations (13.05\%). The shoulders and knees are the most affected anatomical sites. The chi- square tests showed that the diagnosis has significant relevance over the influence and definition of the injuries' treatment $(\mathrm{p}=0.001)$.The diagnosis had statistical significance in relation to the racers' return to sports with the same physical level prior to the injury ( $\mathrm{p}=0.001)$. Conclusion: The shoulders were the most affected by injuries and the fractures the most common lesion in Motocross practice and because of its high impact nature, Motocross has a high rate of injuries.
\end{abstract}

\section{Key words}

Injuries, sporting injuries, sports medicine, physical activity.

* Academic, School of Physical Therapy. Estácio de Sá University, Cabo Frio. Rio de Janeiro, Brasil.

** Professor Esp. Physical Therapist, School of Physical Therapy. Estácio de Sá University, Cabo Frio. Rio de Janeiro, Brasil.

*** Professor M.Sc. Physical Therapist, School of Physical Therapy. Estácio de Sá University, Cabo Frio. Rio de Janeiro and State University of Rio de Janeiro (PPGCEE - UERJ), Brasil.

***** Professor Ph.D. Physical Education, School of Physical Therapy. Estácio de Sá University, Cabo Frio. Rio de Janeiro and State University of Rio de Janeiro (PPGCEE-UERJ), Brasil. 


\section{INTRODUCCIÓN}

El motocross es uno de los deportes más populares y practicados en el mundo. También es considerado como uno de los más peligrosos ya que se ha convertido en la causa de varios accidentes con lesiones significativas para sus practicantes (Heggie \& Carnie, 2012). El conocimiento de las características del motocross y la experiencia en el campo son elementos claves para comprender los mecanismos de lesión y los sitios más afectados en los deportistas.

Las carreras de motocicletas generalmente se clasifican en carreras de circuito de pista y a campo traviesa. Hay diversas variantes del deporte, tales como el motocross. Esta modalidad es practicada al aire libre en largas pistas de tierra, barro y/o arena, con una mezcla de obstáculos naturales y artificiales, utilizando motocicletas todoterreno (off-road). La modalidad también tiene variaciones, como el supercross, que generalmente es practicado por los atletas de motocross debido a las similitudes entre ellos (Grange y Bodnar, 2009).

La modalidad es una de las más populares en el mundo y la competencia todoterreno es una actividad cada vez más popular (Villegas, Bowman, Zoog, Scoot, Haut, Stevens et al., 2015). Los obstáculos característicos de la pista de motocross pueden alcanzar los veinte metros de longitud y las carreras pueden tener hasta cuarenta corredores participando (Gobbi, Francisco, Tuy y Kvitne, 2005).

Debido a que es un deporte de alto impacto, el motocross plantea riesgos para sus atletas, por lo que deben usar una gama de equipos de protección como casco, botas, chaleco, gafas protectoras, rodilleras, coderas y peto, además del uniforme básico que consiste en guantes, chaqueta y pantalones especiales. Todo el equipo tiene como objetivo proteger los órganos vitales de accidentes, las rocas y arcilla lanzadas contra el piloto por la tracción de las motocicletas que se encuentran delante. También tiene como objetivo permitir una buena ventilación y transpiración, usualmente hecho de material liviano y resistente para no afectar el rendimiento del motociclista durante la carrera, lo que le permite una buena movilidad y movimiento en la motocicleta mientras lo mantiene protegido (Gobbi, Tuy y Panuncialman 2004).

Las carreras de motocross están separadas por categorías definidas por la edad de los pilotos y la potencia del motor de la motocicleta. Las categorías son: mini motocicletas de $50 \mathrm{cc}$, $65 \mathrm{cc}, 85 \mathrm{cc}, 125 \mathrm{cc}$, las categorías principales MX2 (250cc) y MX1 (450cc), las categorías de veteranos MX3 (+30) y MX4 (+40) y la categoría femenina WMX.

Las carreras usualmente tienen una duración de 35 minutos más dos vueltas en las categorías principal y veterano. En las categorías niños, la duración es de 20 minutos más dos vueltas, siguiendo las reglas reevaluadas y determinadas para la temporada 2014 (CBM, 2014; FIM, 2014).

El motocross exige mucho físicamente de sus practicantes, los que requieren un alto nivel de acondicionamiento físico para sostener el peso de la motocicleta y absorber los golpes del terreno áspero de las pistas (Konttinen, Kkinen e Inen, 2007). Las áreas más utilizadas para este sustento y absorción son el cuello, la sección media, los brazos y las piernas y es por esto que los corredores profesionales pasan por un régimen diario de entrenamiento físico y técnico para prepararse para las competencias. A pesar del entrenamiento intensivo, la experiencia y el uso del mejor equipo de protección para el piloto, las investigaciones recientes en motocross y carreras muestran un riesgo relativamente alto de lesiones graves (Mcintosh, \& Shea, 2011).

Aunque la competencia de motocicleta todoterreno es una de las actividades deportivas más populares, practicada por millones de personas en todo el mundo, es necesario realizar más estudios sobre los estándares de traumatismos relacionados con el motocross y su prevención (Gorski, Gorski, Mcleod, Suh, Cordeiro, Essien, Berry \& Dada, 2003.

Por lo tanto, el objetivo del estudio fue determinar la tasa de lesiones en los deportistas profesionales de motocross de diferentes categorías, compitiendo a nivel estatal, nacio- 
nal e internacional y evaluar las lesiones más comunes entre los deportistas de motocross y las regiones anatómicas más frecuentemente afectadas por la práctica del deporte.

\section{METODOLOGÍA}

Esta investigación de campo se centró en 45 deportistas, cuyas edades oscilan entre 16 y 46 años, hombres y mujeres, de diferentes nacionalidades y lugares de nacimiento, que compiten en carreras del Campeonato Brasileño de Motocross, Campeonatos Estatales y Campeonatos Internacionales, que respondieron un cuestionario adaptado (Carazzato, Cabrita \& Castropil, 1995).

Se enviaron cuarenta y cinco cuestionarios por correo electrónico y redes sociales a todos aquellos que estuviesen interesados en participar en la investigación. El cuestionario fue autoadministrado y se envió un texto explicativo para las preguntas y el grado de las respuestas. En caso de dudas, los deportistas podrían ponerse en contacto con los investigadores personalmente, por teléfono móvil, Skype o correo electrónico para obtener más aclaraciones.

El cuestionario contenía: fecha de nacimiento, edad en la que el individuo comenzó a practicar motocross, tiempo de práctica del deporte, categoría actual, nivel de competencia, programa de entrenamiento habitual, la práctica de otros deportes, lesiones sufridas durante el entrenamiento o competencia, edad en el momento de la lesión, cómo ocurrió, el tipo de lesión, el tipo y tiempo de tratamiento y cómo fue el regreso a la práctica del deporte. Se consideró como una lesión, cuando hubo un diagnóstico médico que confirmaba la condición. En el caso de los corredores extranjeros que no dominaron el idioma portugués, el cuestionario adaptado también se tradujo al inglés.

Todos los deportistas fueron orientados sobre la investigación, y para participar firmaron una Declaración de Consentimiento Informado de acuerdo con las regulaciones de la resolución brasileña número 466/2012.
Los corredores menores de edad tenían autorización de los padres, quienes firmaron una Declaración de Consentimiento Informado preparada especialmente para menores. Para los deportistas extranjeros, la Declaración de consentimiento informado se adaptó al idioma inglés. El trabajo fue aprobado por el comité de ética de la Universidad Estacio de Sá con el número CAAE: 58003716.6.0000.5284

Se calcularon medidas descriptivas para todas las variables. El chi-cuadrado $(\chi 2)$ se utilizó para analizar las posibles asociaciones de frecuencia entre las variables de estudio. El nivel de significancia se estableció en $5 \%$.

\section{RESULTADOS}

Del total de 45 (cuarenta y cinco) cuestionarios enviados a los deportistas, todos fueron respondidos. Entre los 45 deportistas entrevistados, dos (2) eran extranjeros y los otros cuarenta y tres (43) eran brasileños. Entre el número total de deportistas, cuatro eran mujeres $(8,8 \%)$ y cuarenta y uno $(91,2 \%)$ eran hombres, lo que demuestra que la mayoría de los corredores entre los deportistas de motocross son hombres.

La edad promedio de los atletas fue de 26.7 (+ 9.97), lo que demuestra que los que respondieron eran adultos jóvenes. En términos de edad en la inscripción en motocross, el promedio es de 9.2 (+ 5.02) años, lo que indica un comienzo temprano en el deporte. Con respecto al período de competencia, las cifras indican que los corredores tenían, en promedio, 16.5 años de carrera profesional. Los datos señalan que la mayoría de los corredores que compiten a nivel nacional también compitieron en los niveles estatales.

La Figura 1 muestra la distribución de los atletas según las categorías de competencia. 


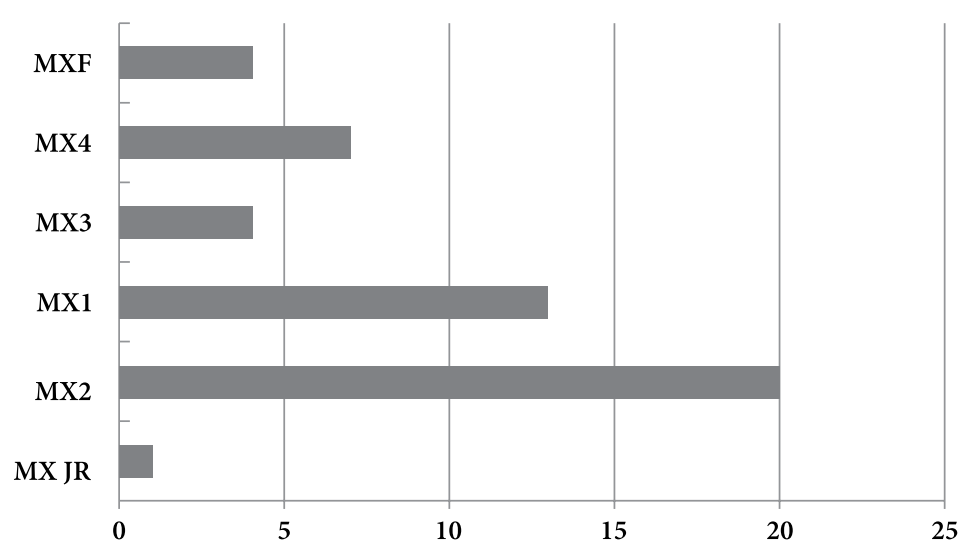

Figura 1. Distribución de los atletas de acuerdo con las categorías de competición.

Entre las categorías de competencia, solo el $1 \%$ de los 45 atletas compitieron en la categoría MXJunior, el 44\% de la muestra compite en la categoría MX2, el $28 \%$ en la categoría MX1, el $8 \%$ en la MX3, el $15 \%$ en la MX4 y el $8 \%$ en las categorías MXF. La categoría MX2 registró la mayor cuantía de atletas, con más relevancia en la muestra con un total de 20 corredores, mientras que MXJunior registró la menor cantidad de atletas (1\%) en la encuesta. La categoría MX1 mostró la segunda mayor suma de atletas con un total de 13 corredores en la muestra.

Hay varias áreas afectadas durante la práctica de motocross. La Figura 2 muestra estas áreas.

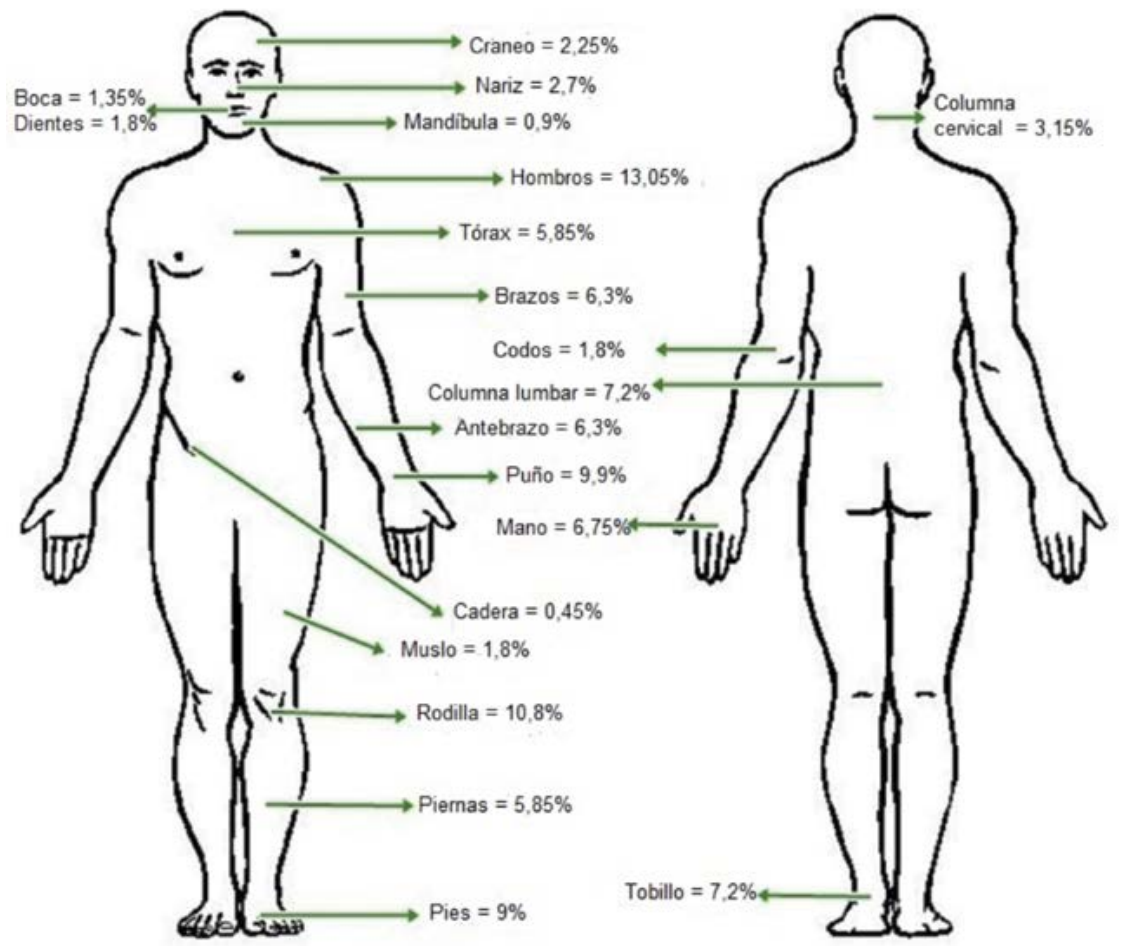

Figura 2. Áreas afectadas durante la práctica de motocross. 
Entre las áreas del cuerpo afectadas por lesiones, cinco (5) regiones resaltaron. Los hombros fueron los más afectados por las lesiones en la práctica de motocross (13.05\%), seguidos por las rodillas (10.8\%), las muñecas $(9.90 \%)$, los pies $(9.0 \%)$ y los tobillos, con $7.20 \%$.

Entre las lesiones más comunes, el énfasis estaba en las fracturas, con un porcentaje de $17.55 \%$, las luxaciones fueron las segundas lesiones más comunes con $13.05 \%$, mientras que otros tipos de lesiones (hematomas, abrasiones, ampollas, mialgias) representaron el 9.45\% de las respuestas en los cuestionarios

Las lesiones entre los deportistas durante las competencias, representa una mayor tasa de incidencia con un total de 152 lesiones entre los corredores que participaron en este estudio. El cuestionario indicó una alta tasa de fracturas (75) y dislocaciones (28) entre los diagnósticos de las lesiones. También presentó las cirugías (23), reposo (22) y la fisioterapia asociada a la cirugía (22) como los tratamientos más frecuentes. Con respecto al regreso a los deportes, los resultados mostraron una alta frecuencia de los atletas con retorno en el mismo nivel físico antes de la lesión, después de los tratamientos (88). Entre las áreas más afectadas por las lesiones, los hombros (34) fueron la región anatómica más afectada seguida de las rodillas (21).

La probabilidad de lesiones entre los atletas durante la práctica, representa una mayor tasa de incidencia con un total de 90 lesiones. Durante la práctica y la competencia, la lesión más frecuente fue la fractura (40) y las luxaciones (14), entre los diagnósticos de las lesiones. El reposo (18) y la cirugía (11) fueron los diagnósticos más frecuentes. Con respecto al regreso a los deportes, los resultados mostraron una alta frecuencia de los atletas que regresaron en la misma forma física previa a la lesión, después de los tratamientos (59). Entre las áreas más afectadas por las lesiones, los hombros (15) fueron la región anatómica más afectada seguida por las rodillas (11).

A pesar de las diferencias observadas entre la cantidad de lesiones sufridas durante la competencia y el entrenamiento, ambas mos- traron una alta tasa de aflicciones causada por la práctica de este deporte.

La Tabla 1 muestra la asociación entre el tratamiento realizado y el retorno a las actividades deportivas; entre el diagnóstico y el tratamiento y entre el diagnóstico y el regreso a los deportes.

\section{Tabla 1}

Prueba de Chi-cuadrado.

\begin{tabular}{lcc}
\hline & $\chi^{2}$ valor & valor $p$ \\
\hline Diagnóstico X Tratamiento & 155.973 & 0.001 \\
\hline Diagnóstico X Regreso & 70.017 & 0.001 \\
\hline Tratamiento X Regreso & 42.661 & 0.121 \\
\hline
\end{tabular}

$\chi^{2}$ : Chi-cuadrado.

La Tabla 1 muestra estadísticamente que el diagnóstico tiene una relevancia significativa sobre la influencia y la definición del tratamiento de las lesiones. El diagnóstico tuvo relevancia estadística en relación con el regreso de los corredores a los deportes con el mismo nivel físico previo a la lesión. No pudimos encontrar una asociación estadística entre los tipos de tratamientos y su influencia en el regreso de los atletas al deporte.

\section{DISCUSIÓN}

La mayoría de los autores (Ascenção, Azevedo, Ferreira, Oliveira, Marques \& Magalhães, 2008; Tomida, Hirata, Fukuda, Tsujii, Kato, Fujisawa \& Uchida, 2005) de los artículos específicos consultados para esta investigación enfatizan la importancia de las lesiones del sistema locomotor en la epidemiología de las lesiones deportivas, en particular el motocross, como un recurso valioso para el desarrollo de programas preventivos y / o terapéuticos. En nuestro trabajo encontramos la misma información, con una alta tasa de lesiones en los miembros superiores y las extremidades inferiores.

En un estudio realizado el 2003, los autores concluyeron que los ejercicios de alta intensidad que se realizan en las carreras de moto- 
cross y el esfuerzo físico específico del deporte debido a la práctica deportiva en terrenos desiguales de barro, arena y tierra, los saltos altos, las curvas y el impacto de los saltos, inducen cambios funcionales que pueden resultar en la disminución del rendimiento físico del corredor (Colburn \& Meyer, 2003). Esto es corroborado por la alta tasa de lesiones observadas en esta investigación.

En este estudio, un pequeño margen de atletas informó que el regreso a las prácticas después del tratamiento de las lesiones fue peor que antes de la lesión, mientras que la mayoría de los atletas informaron regresar al mismo $(88 \%)$ o mejor nivel que (53\%) antes de la lesión. La tasa de corredores que regresaron al mismo nivel antes de la lesión tiene un mayor impacto estadístico tanto en las lesiones sufridas durante el entrenamiento y como en las competiciones.

En un estudio del 2005 que analizó la incidencia de "Lesiones en las carreras de motos en Japón", se detectó que los corredores de motocross son los más afectados por lesiones entre los atletas de motociclismo, donde las fracturas son las lesiones más comunes entre estos deportistas, seguidas de lesiones a los ligamentos y dislocaciones. Este estudio no encontró una correlación del riesgo de lesión entre la edad, la experiencia o el nivel de competencia (Tomida et al., 2005). En nuestra investigación, también encontramos que las fracturas son los tipos más comunes de lesiones entre los competidores de motocross, en una proporción de $17.55 \%$, siendo las luxaciones la segunda incidencia más alta, con una tasa de $13.05 \%$.

En un análisis prospectivo de lesiones en motociclismo todoterreno (Colburn et al., 2903). celebrado durante cuatro rondas de una competencia internacional de Enduro, utilizando la Puntuación de Gravedad de la Lesión (ISS) y la Escala Abreviada de Lesiones (AIS), los autores examinaron a los corredores principiantes en la competencia con el puntaje de lesiones más alto (54\%) de la muestra. Entre las regiones anatómicas más afectadas fueron las extremidades (57\%) y los pies y tobillos los más afectados por las fracturas (36\%). Sin embargo, las lesiones de ligamentos tuvieron la mayor incidencia $(47 \%)$ y las fracturas y dislocaciones fueron las segundas dentro de la prevalencia de las lesiones (46\%).

Con los resultados de esta investigación, encontramos que, de todas las articulaciones del cuerpo humano, la articulación del hombro (13.05\%) es la más abusada y creemos que esto se debe al hecho de que el motocross es un deporte extremo en el que los corredores usan esta articulación constantemente para absorber los impactos del terreno irregular, los hoyos, las secciones de las olas, los saltos o el propio peso de la motocicleta. Además, de acuerdo con el informe de los corredores, durante las caídas, en la mayoría de los casos el hombro es la región que golpea el suelo primero, absorbiendo el impacto del peso corporal añadido al equipo y, dependiendo de la caída, esto no puede evitarse.

A pesar del uso obligatorio de la rodillera como equipo de protección, nos dimos cuenta de que la articulación de la rodilla también se ve muy afectada, representando una proporción del $10.8 \%$ de las áreas afectadas por las lesiones. Al igual que los hombros, las rodillas también forman parte del conjunto de amortiguación de impacto del motocross.

Según informaron los atletas, de acuerdo con el mecanismo de las lesiones que afectan a esta región anatómica, la rodilla está constantemente sujeta a colisiones directas con el suelo durante las curvas debido a la flexión de la cadera, causada por la extensión de la pierna para mejorar la eficiencia al tomar las curvas y el equilibrio, dejando a la región también susceptible de romperse debido al fuerte impacto de los pies en el suelo contra la aceleración de la motocicleta.

Recientemente en 2014, el estudio "Guerrero de Fin de Semana: Hechos o ficción para un traumatismo?" observó una mayor tasa de lesiones en motocross en comparación con otras prácticas deportivas recreativas. Los atletas recreativos deben ser conscientes de los riesgos de lesiones graves durante sus actividades de fin de semana y de que es necesario usar equipo de protección como chaleco, casco, guantes 
y botas para practicar este deporte (Roberts, Quellet, Mcbeth, Kirkpatrick, Dixon \& Ball, 2014).

En nuestro estudio, demostramos que incluso con todo el equipo de seguridad obligatorio y el entrenamiento diario específico de los atletas, la incidencia de lesiones en la práctica profesional de motocross sigue siendo alta, como en la práctica amateur.

De acuerdo con un estudio que compara las lesiones entre los motociclistas todoterreno y en pista (o ciclistas callejeros) (Grange, Corbett \& Cotton, 2004) aunque ambos estilos presentaron lesiones más similares en el análisis de los registros de un hospital regional en California, las posibilidades de un traumatismo abdominal, un traumatismo de tórax y un traumatismo cutáneo o riesgo de muerte fueron mucho más altos para los motociclistas callejeros que para los corredores todoterreno. Esto se observó en nuestro estudio en el que diagnosticamos un porcentaje bajo de lesiones en la cabeza y traumatismo torácico y abdominal entre los corredores de motocross. Sin embargo, las lesiones cutáneas fueron relevantes y consistieron en lesiones deportivas de rutina según los informes de los atletas.

En un análisis de la relación con las lesiones y la edad de los corredores (Mullin, Jackson, Langley \& Norton, 2000) se encontró que hay pocas pruebas de que los años de experiencia en la conducción reduzcan el riesgo de lesiones moderadas a lesiones fatales causadas por accidentes de motocicleta. Sin embargo, después de analizar los datos recopilados, los autores diagnosticaron que los pilotos jóvenes, especialmente los menores de 20 años, tienen un alto riesgo de lesiones en comparación con los ciclistas mayores, concluyendo que la familiaridad con las características distintivas de la motocicleta es la única medida de experiencia asociada con un efecto protector.

También observamos con esta investigación que entre las 45 personas entrevistadas, la mayor concentración de atletas se encuentra en las categorías MX2 (44\%) y MX1 (28\%), en la medida en que la muestra incluye atletas de edades comprendidas entre 18 y 30 años, comparada a las categorías de veteranos de atletas mayores de 30 años, MX3 (8\%) y MX4 (15\%). En nuestra muestra, los adultos jóvenes tuvieron una diferencia estadísticamente más significativa, mostrando una mayor incidencia de lesiones que la mayoría de los atletas experimentados. Esta información es similar a los resultados del estudio de 35 deportes a nivel nacional en Suecia (Aman, Forssblad \& Henriksson-Larsen, 2016).

Teniendo en cuenta la falta de literatura específica en portugués sobre motocross para la base de datos bibliográfica de este estudio, buscamos estudios en el extranjero sobre la incidencia de lesiones (Duthon, Masoure \& Mentrey, 2015) en motociclismo todoterreno y en la práctica de otras modalidades para fundamentar este trabajo.

Para futuras investigaciones, el estudio puede separarse por edad, ya que la literatura (Dick, White \& Bopf 2014; Singh, Malhotra \& Kyle, 2015) hay se ha centrado en las lesiones sufridas por niños y adolescentes en la práctica de motocross. Entonces, sería posible comparar los datos en Brasil con los hallazgos en otros países.

\section{CONCLUSIONES}

El motocross presenta un alto riesgo de lesiones. Con esta investigación, también notamos que los corredores de edades comprendidas entre 18 y 30 años, compitiendo en las categorías MX2 y MX1, tienen un mayor riesgo de lesiones que la mayoría de los atletas experimentados, registrando el mayor número de lesiones de la muestra. El deporte también tiene un predominio de deportistas masculinos. Los atletas se unen a la práctica deportiva temprana, a una edad promedio de 9 años y tienen un promedio de actividad profesional de 16 años. Los hombros y las rodillas son las regiones anatómicas con mayor incidencia de lesiones, y las fracturas, dislocaciones y contusiones son los tipos más comunes.

La cirugía, el reposo y la fisioterapia son los tratamientos más adoptados por los corredores de motocross estudiados, y la mayoría de 
los participantes informaron que reanudaron sus actividades deportivas en el mismo nivel físico en el que se encontraban antes de la lesión.

Creemos que este trabajo puede ayudar a fomentar más estudios sobre el tema, teniendo en cuenta que debido a la escasez de investigación con este deporte, este estudio muestra datos importantes en cuanto a la referencia del perfil epidemiológico del motocross. Esta investigación puede proporcionar una dirección para el desarrollo de programas preventivos y orientación para los atletas y profesionales involucrados en esta modalidad.

\section{REFERENCIAS BIBLIOGRÁFICAS}

Aman, M., Forssblad, M., Henriksson-Larsen, K. (2016). Incidence andseverity of reported acute sports injuries in 35 sports using insurance registry data: Incidence of acute sports injuries. Scand J Med Sci Sports, 26(4):451-62

Ascenção, A., Azevedo, V., Ferreira, R., Oliveira, E., Marques, F., Magalhães, J. (2008). Physiological, biochemical and functional changes induced by a simulated 30 min off-road competitive motocross heat. The Journal of Sports Medicine and Physical Fitness, 48(3):311-319.

Carazzato, J.G., Cabrita H., Castropil, W. (1996). Repercussão no aparelho locomotor da prática do judô de alto nível. Revista Brasileira de Ortopedia, 31(12) dezembro.

Colburn, N.T., Meyer, R.C. (2003). Sports injury or trauma? Injuries of the competition off-road motorcyclist. Injury International Journal of the Care of the Injured. 34:207- 214.

Confederação Brasileira de Motociclismo (2014). Regulamento 2014 do Campeonato Brasileiro de Motocross. Cidade sede da instituição. Available on http:// cbm.esp.br/sistema/regulamentos/ f191ca3c096389bb595a696117c7a 46a. pdf. Acesso em 12/04/2014.
Dick, C.G., White, S., Bopf, D. (2014). A review of the number and severity of injuries sustained following a single motocross event. Journal of Orthopaedics, 11(1): 23-27.

Duthon, V., Masoure, P., Mentrey, J. (2015). Epidemiology and Medical Coverage of Motocross and Supercross Races. Sports Injury. 2825-2833.

Fédération Internationale de Motocyclisme. (2014). Regulamento 2014 do Campeonato Mundial de Motocross MXGP, MX2 e WMX. Available on http://www.fim-live.com/fileadmin/ alfresco/6520001_eng.pdf. Acesso em $12 / 04 / 2014$

Gobbi, A., Tuy, B., Panuncialman, I. (2004). The Incidence of Motocross Injuries: a 12-year investigation - Knee Surgery, Sports Traumatolology, Arthroscopy. 2: 574-580.

Gobbi, A.W., Francisco, R.A., Tuy, B., Kvitne, R.S. (2005). Physiological characteristics of top level off-road Motorcyclists. British Journal of Sports Medicine. 39: 927- 931.

Gorski, T.F., Gorski, Y.C., Mcleod, G., Suh, D., Cordeiro, R., Essien, F., Berry, D., Dada, F. (2003). Patterns of Injury and Outcomes Associated with Motocross Accidents. The American Surgeon. 69:895-898.

Grange, J.T., Bodnar, J.A., Corbett, S.W. (2009) Motocross medicine. Current Sports Medicine Reports, 8(3):125-130.

Grange, J.T., Corbett, S.W., Cotton, A. (2004). Street Bikes Versus Dirt Bikes: A Comparison of Injuries Among Motorcyclists Presenting to a Regional Trauma Center. The Journal of Trauma Injury, Infection, and Critical Care, 57(3): 591-594. 
Heggie TW, Carnie DJ. (2012) Epidemiology of injury of adventure and extreme sports. The Epidemiology of Injury in ATV and Motocross Sports. Medicine and sports science. 58:158-172.

Konttinen T, Kkinen KH, Inen HK. (2007). Cardiopulmonary loading in motocross riding. Journal of Sports Science, 25(9): 995-999.

Mcintosh A, Shea K. (2011). ATV and Motocross Sports. Sports Medicine Update. $2-6$.

Mullin B, Jackson R, Langley J, Norton R. (2000). Increasing age and experience: are both protective against motorcycle injury? A case-control study. Injury Prevention. 6:32-35.
Roberts DJ, Quellet JF, Mcbeth PB, Kirkpatrick AW, DixonE, Ball CG. (2014). The "weekend warrior": Fact or fiction for major trauma? Canadian Journal of Surgery, 57(3): E62-8.

Singh R, Malhotra A, Kyle N, Hay S. (2015). An epidemiological study of paediatric motocross injuries in the United Kingdom. J Child Orthop. 9: 385- 390.

Tomida Y, Hirata H, Fukuda A, Tsujii M, Kato K, Fujisawa K, Uchida A. (2005). Injuries in elite motorcycle racing in Japan. British Journal of Sports Medicine. 39:508-511.

Villegas CV, Bowman SM, Zoog CK, Scoot VK, Haut ER, Stevens KA, et al. (2015). The hazards of off-roas motor sports: Are four wheels better than two? Aug 21(15): 473-8.

\section{Dirección para correspondencia}

Flavio Boechat de Oliveira

Academic School of Physical Therapy

Estácio de Sá University, Cabo Frio

Rio de Janeiro - Brasil.

Dirección: Rua Roberto Nogueira Machado, 10 Centro. Cabo Frio. Rio de Janeiro

Código postal: 28907-040

Tel. (22) 26436551

Contacto:

fisioboechat@gmail.com

Recibido: 15-09-2017

Aceptado: 06-11-2017 